\title{
Using Provenance to Improve Workflow Design
}

\author{
Frederico T. de Oliveira ${ }^{1}$, Leonardo Murta ${ }^{1,2}$, Claudia Werner ${ }^{1}$, and Marta Mattoso ${ }^{1}$ \\ ${ }^{1}$ COPPE/ Computer Science Department \\ Federal University of Rio de Janeiro (UFRJ) \\ \{ftoliveira, werner, marta\}@cos.ufrj.br \\ ${ }^{2}$ Instituto de Computação \\ Universidade Federal Fluminense (UFF) \\ leomurta@ic.uff.br
}

\begin{abstract}
With the popularity of scientific workflow management systems (WfMS), workflow specifications are becoming available. Provenance support in WfMS can help reusing third party code. Browsing can be done through queries instead of ad-hoc search on the Web. Finding dependencies among programs or services through provenance queries, without tool support, is not a trivial task. Due to the huge number of program versions available and their configuration parameters, this task may be heavily error prone and counterproductive. In this work we propose a recommendation service that aims at suggesting frequent combinations of scientific programs for reuse. Our recommendation service is designed to work over WfMS that provide provenance on workflow specification and execution logs. We have based our service on software components reuse and data mining techniques, and implemented a prototype with Vistrails WfMS.
\end{abstract}

\section{Introduction}

Workflow management systems are getting more complicated and providing more functions. Each day, more services are available and their combinations become more complex. Scientific workflows are based on the automation of scientific processes in which scientific programs are associated, based on data and control dependencies. Frequently, these scientific programs are third party code, shared by scientists from a common domain. Aiming at reusing these programs, the scientist browses public execution scripts to see how the program should be parameterized to his specific needs. With the popularity of scientific workflow management systems (WfMS), workflow specifications are also becoming available.

Provenance support in WfMS can help reusing third party code. Browsing can be done through queries instead of ad-hoc search on the Web. Despite that, reusing a scientific program often involves reusing complementary programs. Finding dependencies among programs or services through provenance queries, without tool support, is not a trivial task. Due to the huge number of program versions available and their configuration parameters, this task may be heavily error prone and counterproductive. Automating those tasks can reduce errors and improve reliability. Even if a powerful 
workflow provenance support is provided, such as the management of workflow versioning of Vistrails [1], identifying adequate combinations can be time consuming and may involve designing complex queries. Current e-Science infrastructures provide the capability to combine services from a diverse set of providers in a variety of ways. However, they can only be exploited by a minority of specialists who are familiar with workflow composition systems, programming paradigms, distributed infrastructures and complex problem solving environments [2]. In this work we propose a recommendation service that aims at suggesting frequent combinations of scientific programs for reuse. Our recommendation service is designed to work over WfMS that provide workflow specification databases or workflow execution logs. We have based our service on software components reuse and data mining techniques, and implemented a prototype that works with Vistrails.

Our prototype consists on a recommendation system based on a collaborative filtering approach [3]. This recommendation system let users discover useful workflows components and how they can be combined. According to collaborative filtering approach, collected provenance histories are used to recommend a set of candidate services that may be useful to individual scientists.

This work is organized as follows. In section 2, we present the background of our research, containing an overview of workflow and some related work and related techniques. We describe our approach in section 3 and the usage of our prototype in section 4. Finally, we conclude our work presenting some advantages of recommendation in workflow design and some future work in section 5.

\section{Background}

The scientific workflow design process still occurs in an ad-hoc manner, driving to irreproducible results due to absence of predefined processes or methods. In some cases, the workflow is not explicit because the scientists directly connect programs to perform the experiment, hindering the comprehension of the whole process. In other cases, despite the existence of a WfMS that manages the interaction of the programs and services necessary to perform the experiment, no systematic method is used throughout its design.

In this section we present an overview on the benefits component-based workflow, software reuse and component-based software development, and collaborative filtering can provide to workflow design.

\subsection{Software Reuse and Component-Based Software Development}

Software reuse is the process of creating software systems from existing software [4]. It encompasses two main perspectives, which are development for reuse and development with reuse. Software development for reuse aims to produce assets that can be reused later. On software development with reuse, assets are coupled to the system under development. Reusable assets represent any product derived from software development, for example, source code, components, test cases, etc. 
Component-based software development is a technique for software reuse that focus on reusing well defined components, produced via an independent process [5]. Component-based software development uses components, interfaces and connectors as first-class entities to structure software systems. Components, which are reusable assets [6], make use of interfaces, described in a contractual manner, to interact with the remaining software elements [7]. Connectors are responsible for performing the binding among components.

When component-based software development is in place, the software development teams can be classified by their roles in the process. Some teams are in charge of developing components. These teams, named producers, produce reusable components that serve to others. Other teams, named consumers, are in charge of developing systems by reusing existing components. Finally, there are some hybrid teams, which act as both producers and consumers. They reuse existing components to produce more other components.

The component-based software development process is currently supported by a variety of methods. The most well known and adopted are Catalysis [6], UML Components [8], and KobrA [9]. However, besides the existence of these methods, components must be sufficiently widespread to allow their reuse and composition in different contexts, as it is expected from workflow tasks and services [10].

There are already some exiting work that apply reuse to the conception of workflows. De Roure and Goble [2] address several issues to promote workflow reuse. One of them is the recommendation of workflow and services, which is a vital part of enabling sharing through discovery by other scientists and it is also a part of communicating know-how. For instance, Taverna has made over 3500 bioinformatics orientated operation available to its users, and it would probably get benefits from recommendation systems.

\subsection{Component-Based Workflow}

Zhuge [11] proposes an approach to the development of component-based workflow system through integrating the characteristics of software component. On this approach, the user combines workflows components, which are business process units, to build a complete business process. Workflow components are defined as a workflow process that describes a category of complete business process units, Compared with traditional workflow development, the component-based workflow system has the following potential advantages [11]:

- Lower complexity: a complex workflow can be transformed to a workflow component hierarchy, where each workflow component has the lower complexity than the whole workflow;

- Reusability: a workflow component can be safely reused by any other workflow component or tasks through its access interface;

- Adaptability: component modifications or new component additions will not influence the other components;

- Connectivity: different components can be easily connected; 
- Maintainability: components can be maintained at run time if the maintenance can be finished before its execution, increasing the adaptability of the workflow;

- Error localization: workflow definition errors and execution errors occur within components, enabling the checking mechanism to localize them; and

- User's acceptability: workflow components encapsulate domain business process units, so it can be more easily checked and used.

These advantages have a business scenario in mind. However, they may be extended to scientific experiments also. For example, Vistrails components are called modules and Kepler components are called actors.

Despite the documentation available through provenance, it is not systematically used in the design of scientific workflows. On section 3, we expose how our approach can use provenance to help workflow design.

\subsection{Recommendation Systems and Collaborative Filtering}

Recommendation systems apply data mining techniques to the problem of helping user find the items they would like to purchase at E-Commerce sites by producing a predicted likeliness score or a list of the top recommended items for a given user [12].

The Collaborative Filtering is considered a key technology of recommendation systems, which provide the user with a set of candidate items that may be useful or preferable to the individual user, from a large amount of items [3]. In other words, collaborative filters help people make choices based on the opinions of other people.

For example, in the e-commerce scenario, if a customer searches for the book "The Secret" at an online store, the store recommends other products, like The Secret: 2008 Day-to-Day Calendar, The Secret Soundtrack, etc.

We can also map some concepts adopted in e-commerce domain into concepts concerned to scientific domain. Table 1 illustrates those mapping.

Table 1. E-commerce concepts mapped into scientific experiments concepts

\begin{tabular}{|c|l|l|l|l|}
\hline Domain & \multicolumn{4}{|c|}{ Concepts } \\
\hline E-commerce & Customer & Cart & Product & Preference \\
\hline $\begin{array}{c}\text { Scientific } \\
\text { Experiment }\end{array}$ & Scientist & Workflow & Component & Context \\
\hline
\end{tabular}

Similarly, to help scientists design workflows, we propose a tool to recommend services and task that are more likely to be used by the scientist.

\section{Workflow Process Recommendation in Vistrails}

In addition to the reuse of components in isolation, we aim to reuse the most common relations between them, increasing productivity and quality of workflow design. Our recommendation service is designed to work over WfMS that provides provenance on histories of workflow specification and execution logs, as Vistrails WfMS does. Based on the collaborative filtering approach, collected histories are used to recommend the 


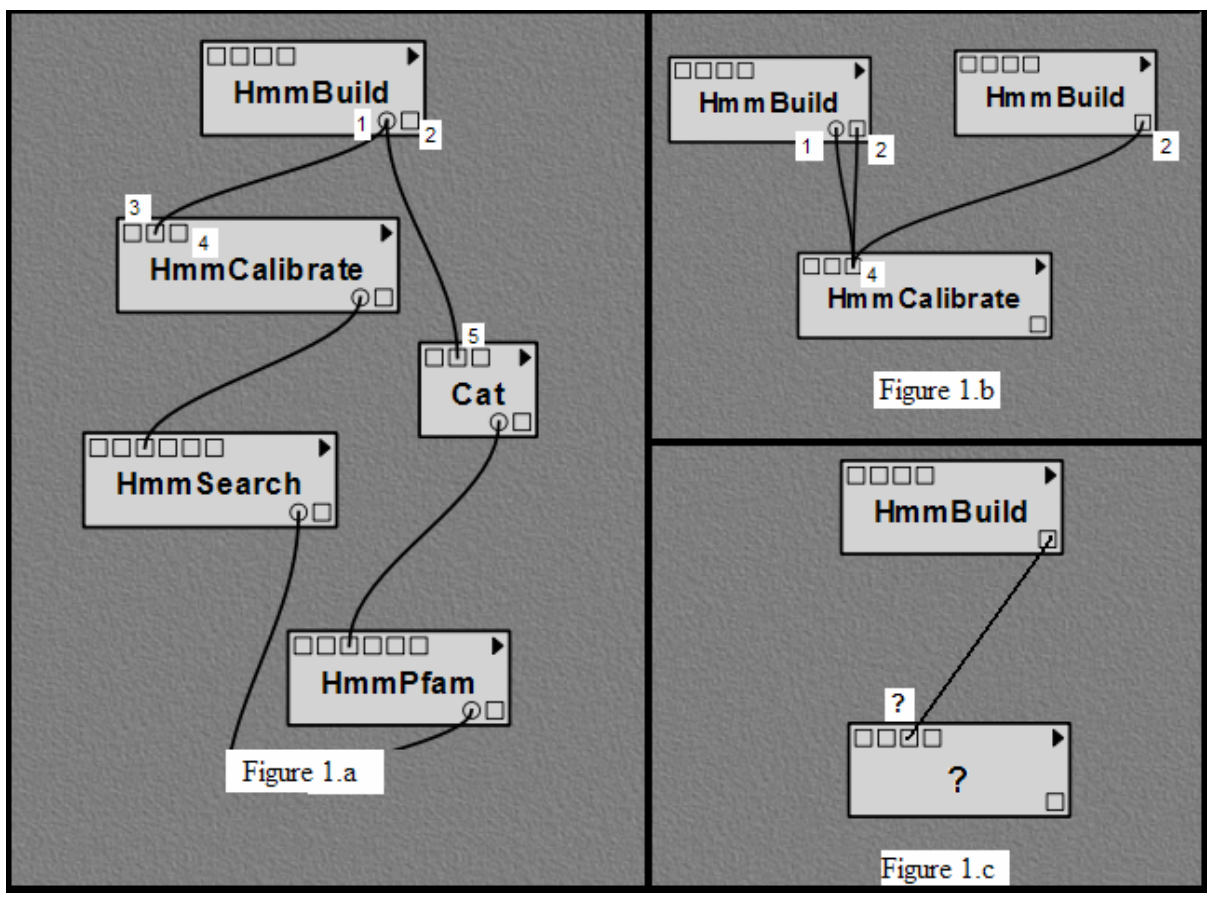

Fig. 1. Component recommendation example in the bioinformatics domain. Ports 1 and 2 are the output ports DestinationDir and StdOut, respectively. Ports 3, 4 and 5 are the input ports SourceDir, HmmPath and Dir, respectively.

scientist a set of candidate components that may be useful to the workflow under design. We propose to infer the need of a component and proactively recommend that component to the scientist.

First, we parse the workflows files to extract the relations between two components. Those relations are mapped into a database table, containing the components, the port that they are connected and the workflow itself. Then, each time a scientist adds a component to his current workflow, the tool automatically analyses the database and recommends the most relevant components previously connected to this one, and indicates how they can be connected.

For example, Figure 1 illustrates parts of some existing bioinformatics workflows. These workflows employ the use of some workflow components, such as "HmmBuild", "HmmCalibrate", and "Cat". These components are connected in different ways in these workflows. Assuming that scientists need to conceive new workflows, if they add the component "HmmBuild" to a new workflow, it is possible to automatically infer that "HmmCalibrate" and "Cat" may also be needed in this new workflow. Moreover, it is also possible to detect how "HmmCalibrate" and "Cat" connect to the existing components in the new workflow, based on how they were connected in previous workflows. 
Besides suggesting related workflow components, we can also extract some metrics that provides support to the scientist on selecting the appropriate workflow components. For instance, in the example shown in Figure 1, the recommendation confidence metric regarding connecting the "HmmBuild" StdOut port to HmmPath port from "HmmCalibrate" is $40 \%$. Moreover, the recommendation confidence metric regarding adding component "Cat" after adding component "HmmBuild" is $20 \%$. It is possible to notice that our recommendation confidence metric consists of the conditional probability of selecting a component ("HmmCalibrate" or "Cat", in our example), assuming that another component is also selected ("HmmBuild", in our example).

Finally, in Figure 2 we present the algorithm used to detect the related components and compute the recommendation confidence metric.

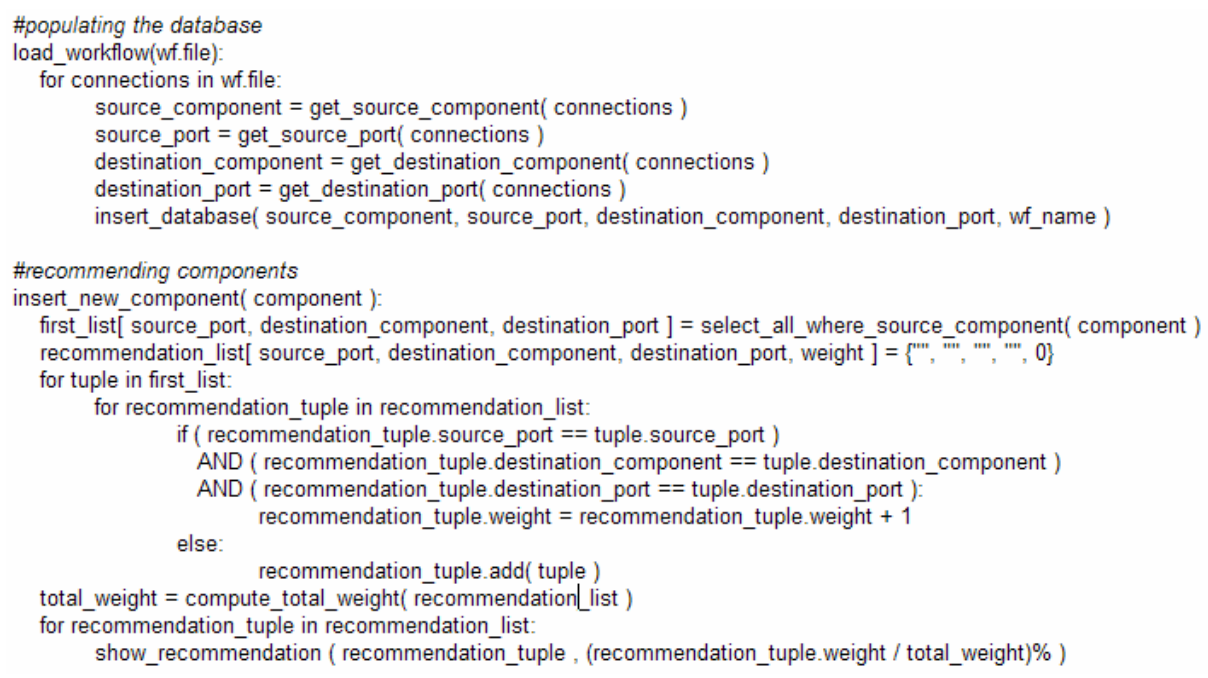

Fig. 2. Algorithm for component recommendation

\section{Usage Details}

Preliminary ideas on these recommendation techniques have been implemented and incorporated to the Vistrails WfMS. By parsing the XML files that store versions of workflows we were able to evaluate and recommend.

Figure 3 illustrates the prototype working inside Vistrails, based on the previously discussed example shown in Figure 1. When the user adds the module HmmBuild to his workflow, the tool automatically recommends two other modules that may be connected to this one: "HmmCalibrate" and "Cat". The first row means that port StdOut of HmmBuild has been connected to port HmmPath of HmmCalibrate in $40 \%$ of previously designed workflows. The recommendations are on the bottom right corner of figure 3 . 


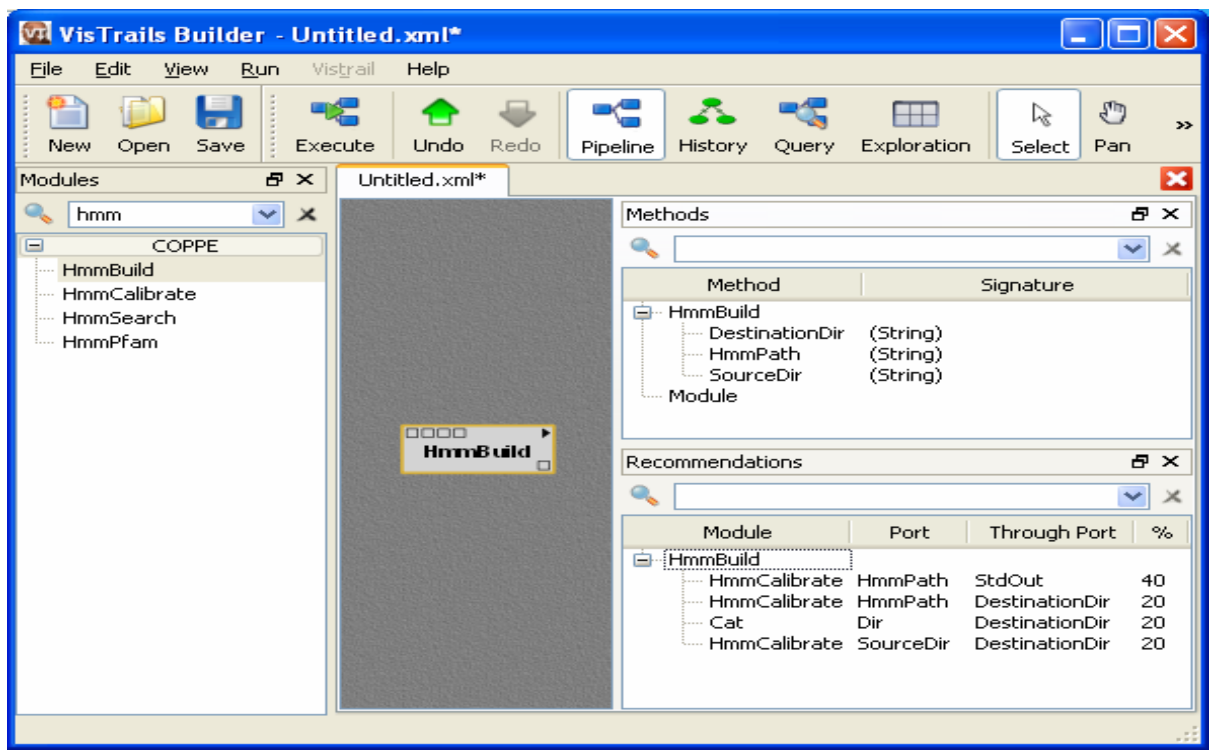

Fig. 3. Recommendation Prototype in Vistrails

\section{Conclusion}

In this paper, we presented a recommendation system for suggesting the reuse of preexisting scientific workflow components during the conception of new workflows. We expect that this approach may help to propagate the benefits of software reuse and component-based development to the context of scientific workflows.

However, we could detect some limitations of our approach. The current version of our prototype recommends only a subsequent component based on previously used connection. Now, we are aiming to improve the approach recommending a component investigating the whole path. We also believe that specifying a context to each workflow will help doing a better recommendation. For instance, if a scientist is designing a bioinformatics workflow, connections that come from others bioinformatics workflows should have a higher weight and then more relevance in the recommendation list. At last, we are considering using the number of workflows executions to establish a higher weight to more used workflows.

As a future work, we intend to analyze other possibilities regarding recommendation algorithms in the next prototype versions and perform some structured evaluations to quantify the benefits provided by our approach.

\section{Acknowledgments}

This work was partially funded by CNPq. 


\section{References}

1. Scheidegger, C., Koop, D., Santos, E., Vo, H., Callahan, S., Freire, J., Silva, C.: Tackling the provenance challenge one layer at a time. Concurrency and Computation: Practice and Experience (2007)

2. De Roure, D., Goble, C.: MyExperiment - A Web 2.0 Virtual Research Environment. In: International Workshop on Virtual Research Environments and Collaborative Work Environments, Edinburgh, UK (May 2007)

3. Sarwar, B.M., Karypis, G., Konstan, J.A., Riedl, J.: Item-Based Collaborative Filtering Recommendation Algorithms. In: Proc. of the 10th International World Wide Web Conference (WWW10), Hong Kong, pp. 285-295 (2001)

4. Krueger, C.W.: Software Reuse. ACM Computing Surveys 24(2), 131-183 (1992)

5. Brown, A.W.: Large Scale Component Based Development. Prentice Hall PTR, Englewood Cliffs (2000)

6. D'Souza, D., Wills, A.: Objects, components, and frameworks with UML: The catalysis approach. Addison-Wesley, Reading (1998)

7. Szyperski, C.: Component Software: Beyond object-oriented programming. AddisonWesley, Reading (2002)

8. Cheesman, J., Daniels, J.: UML Components: A Simple Process for Specifying Component-Based Software. Addison-Wesley, Reading (2000)

9. Atkinson, C., et al.: Component-Based Product Line Engineering with UML. AddisonWesley, Reading (2001)

10. Ludäscher, B.: Scientific workflow management and the Kepler system. Concurrency and Computation: Practice \& Experience, 2006 18(10), 1039-1065 (2006)

11. Zhuge, H.: Component-based workflow systems development. Decision Support Systems 35(4), 517-536 (2003)

12. Hill, W.: Recommending and Evaluating Choices in a Virtual Community of Use. In: Conference on Human Factors in Computing Systems (1995) 